15

\title{
Гидродинамический механизм формирования градиента температуры в тонких нематических пленках
}

\author{
(C) A.B. Захаров \\ Институт проблем машиноведения РАН, \\ Санкт-Петербург, Россия \\ E-mail: alexandre.zakharov@yahoo.ca
}

(Поступила в Редакцию 19 декабря 2016 г.)

\begin{abstract}
Теоретически, в рамках нелинейного обобщения теории Эриксена-Лесли, с учетом уравнения теплопроводности, исследован механизм формирования градиента температуры в изначально равномерно прогретом гибридно-ориентированном жидкокристаллическом (ГОЖК) канале микроскопических размеров под действием стационарного гидродинамического потока. Рассмотрен случай полной термической изоляции одной из поверхностей ГОЖК-канала при условии, что на другой поверхности поддерживается постоянная температура. Показано, что на величину разности температур $\chi_{\max }(\xi)$ по сечению ГОЖК-канала обусловленной действием горизонтального стационарного потока с „треугольным“ профилем скорости $u(z, \xi)$ сильно влияет положение $\xi$ максимального значения скорости. Было показано, что в случае ЖК-системы образованной молекулами 4- $n$-пентил- $n^{\prime}$-цианобифенила гидродинамический поток, характеризующийся положением максимума $\xi=0.98$ величины скорости $u(z, \xi=0.98) \sim 0.9 \mu \mathrm{m} / \mathrm{s}$, формирует максимальную разность температур $\chi_{\max }(\xi)=0.03(\sim 9 \mathrm{~K})$ по сечению ГОЖК-канала.
\end{abstract}

Работа выполнена при финансовой поддержке РФФИ (грант N 16-02-00041a).

DOI: 10.21883/FTT.2017.07.44610.445

\section{1. Введение}

В последнее время изучению влияния внешних воздействий, обусловленных электрическими и магнитными силами, на структурные и динамические свойства жидкокристаллических (ЖК) систем инкапсулированных в микро- и наноразмерные объемы уделяется достаточно много внимания [1-5], в то время как прогресс в понимании физических механизмов, ответственных за воздействие градиентов температуры на релаксационные и гидродинамические свойства таких ЖК-систем, более чем скромный [6-9]. И это не взирая на тот факт, что проблема, связанная с движением ЖК-капли, помещенной между двумя горизонтальными поверхностями и находящейся под действием градиента температуры, впервые привлекла внимание исследователей в начале XX-го века [10]. Позже, в рамках классической теории Эриксена-Лесли [11,12], было показано, что в гибридноориентированной нематической ячейке микроскопических размеров градиент температуры $\nabla T \sim \Delta T / d$, направленный вертикально от нижней ограничивающей поверхности к верхней, инициирует гидродинамический поток ЖК-материала в горизонтальном направлении [6]. Величина скорости этого потока $v \sim \frac{d}{\eta} \sigma_{z x}^{t m}$, где $d-$ толщина ЖК-ячейки, $\Delta T=T_{\text {up }}-T_{\text {lw }}-$ разность температур на ограничивающих поверхностях, $\eta-$ вязкость ЖК-системы, $\sigma_{z x}^{t m} \sim \xi \frac{\Delta T}{d^{2}}-$ тангенциальная компонента термомеханического тензора напряжения [6], а $\xi-$ термомеханическая постоянная. Экспериментальные исследования гидродинамического потока в горизонтальном ЖК-канале позволили оценить термомеханическую постоянную $\xi \sim 10^{-12} \mathrm{~J} / \mathrm{K} \cdot \mathrm{m}$ [7]. Позже было показано, что в случае гибридно-ориентированного ЖК (ГОЖК)-канала, с гомеотропной ориентацией ЖК-молекул на верхней, и планарной на нижней ограничивающих поверхностях, под действием градиента температуры формируется горизонтально направленный гидродинамический поток, с таким „треугольно-подобным“ профилем скорости $v_{\text {eq }}(z)$ по z-сечению ЖК-канала, что максимальное значение $\left(\max v_{\text {eq }}(z)\right)$ достигалось вблизи верхней, более теплой ограничивающей поверхности [8]. В связи с этим естественно возникает вопрос: возможно ли формирование градиента температуры в изначально равномерно прогретом ГОЖК-канале под действием горизонтально направленного ЖК-потока с таким „треугольно-подобным“ профилем скорости, чтобы он характеризовался ярко выраженным максимумом вблизи верхней ограничивающей поверхности? Другими словами, удастся ли таким гидродинамическим потоком разогреть верхнюю гомеотропно ориентированную ограничивающую поверхность и тем самым сформировать градиент температуры, направленный поперек ГОЖК-канала, микроскопической ширины?

Ответ на этот вопрос будет дан в рамках нелинейного обобщения классической теории ЭриксенаЛесли $[11,12]$, с учетом уравнения теплопроводности [8]. Будет рассмотрен такой температурный режим, когда температура на нижней ограничивающей поверхности постоянна $\left(T_{\mathrm{lw}}=\mathrm{const}\right)$, а тепловой поток через верхнюю ограничивающую поверхность равен нулю. Таким образом, разность температур $\Delta T=T_{\text {up }}-T_{1 \mathrm{w}}$, которая в 
начальный момент времени равна нулю, должна достичь максимально возможного значения для данного профиля скорости и в интервале температур соответствующих существованию нематической фазы.

\section{2. Основные гидродинамические уравнения}

Рассмотрим термотропный ЖК в горизонтальном канале микроскопической ширины $d$. Будем считать, что в начальный момент времени ЖК-канал равномерно прогрет таким образом, что температура на обеих ограничивающих поверхностях одна и та же. Будем также предполагать, что верхняя ограничивающая поверхность канала обработана таким образом, что на ней достигается гомеотропная ориентация директора $\left(\hat{\mathbf{n}}_{z=d} \| \mathbf{z}\right)$, в то время как на нижней ограничивающей поверхности достигается планарная ориентация директора $\left(\hat{\mathbf{n}}_{z=0} \perp \mathbf{z}\right)$, и тем самым реализуется ГОЖК-канал. Следует отметить, что система координат выбрана таким образом, что ось $\mathbf{z}$ направлена от нижней границы канала к верхней перпендикулярно обеим ограничивающим поверхностям, ось $\mathbf{x}$ совпадает с направлением директора на нижней ограничивающей поверхности, а ось $\mathbf{y}=\mathbf{z} \times \mathbf{x}$ направлена перпендикулярно обеим осям х и z. Это позволяет нам записать граничное условие для поля директора в виде

$$
\theta_{z=0}=\frac{\pi}{2}, \quad \theta_{z=d}=\pi,
$$

где угол $\theta$ (полярный угол) образован направлением директора $\hat{\mathbf{n}}$ и осью $\mathbf{z}$. Далее будем предполагать, что переориентация поля директора происходит в плоскости XOZ. Принимая во внимание тот факт, что ширина ЖК-канала $d \ll l$, где $l$ - длина канала, можно предположить, что все физические величины, вовлеченные в процесс переориентации директора $\hat{\mathbf{n}}(z, t)=\sin \theta(z, t) \hat{\mathbf{i}}+\cos \theta(z, t) \hat{\mathbf{k}}$, зависят только от пространственной переменной $z$ и времени $t$. Здесь $\hat{\mathbf{i}}$, $\hat{\mathbf{k}}$ - орты пространственных осей $\mathbf{x}$ и $\mathbf{z}$ соответственно, a $\hat{\mathbf{n}}$ - орт поля директора. При отсутствии внешних сил в таком ГОЖК-канале под действием упругих и поверхностных сил устанавливается линейное распределение поля директора, характеризующееся полярным углом $\theta(z)=\frac{\pi}{2 d}(z+1)$ [13]. Предположим далее, что на нижней поверхности канала поддерживается постоянная температура, в то время как верхняя поверхность полностью термически изолирована так, что выполняется условие

$$
\begin{gathered}
T_{z=0}=T_{1}, \\
-\lambda_{\perp}\left(\frac{\partial T}{\partial z}\right)_{z=d}=0,
\end{gathered}
$$

где $\lambda_{\perp}-$ коэффициент теплопроводности ЖК-фазы в направлении перпендикулярном направлению директоpa $\hat{\mathbf{n}}$. Принимая во внимание тот факт, что толщина
ЖК-канала варьируется в пределах нескольких микрометров, будем считать, что плотность ЖК-фазы постоянна по сечению канала $\left(\rho_{m}=\mathrm{const}\right)$. Таким образом, мы имеем дело с несжимаемой ЖК-фазой и условие несжимаемости принимает вид

$$
\frac{\partial v_{x}(z, t)}{\partial x}+\frac{\partial v_{z}(z, t)}{\partial z}=0
$$

Последнее условие с учетом отсутствия проскальзывания на одной из ограничивающих поверхностей ЖК-канала (например, нижней)

$$
v_{x}(z, t)_{z=0}=0, \quad v_{z}(z, t)_{z=0}=0
$$

приводит к тому, что в несжимаемом ГОЖК-канале существует только один гидродинамический поток, направленный параллельно ограничивающим поверхностям [8]

$$
\mathbf{v}=v_{x}(z, t) \hat{\mathbf{i}}=u(z, t) \hat{\mathbf{i}}
$$

Под действием гидродинамического потока в ГОЖКканале возникает упругий

$$
\mathbf{T}_{\mathrm{el}}=-\left[\mathscr{g}(\theta) \theta_{, z z}+\frac{1}{2} \mathscr{G}_{, \theta} \theta_{, z}^{2}(\theta)\right] \mathbf{j}
$$

вязкий

$$
\mathbf{T}_{\mathrm{vis}}=\left[\gamma_{1} \theta_{, t}-\mathcal{A}(\theta) u_{, z}\right] \mathbf{j}
$$

и термомеханический $[6,8]$

$$
\mathbf{T}_{t m}=\xi \theta_{, z} T_{, z}\left(\frac{1}{2}+\sin ^{2} \theta\right) \mathbf{j}
$$

моменты, действующие на единицу объема ЖК-фазы. Здесь

$$
\theta_{, z z}=\frac{\partial^{2} \theta(z, t)}{\partial z^{2}}, \quad \theta_{, t}=\frac{\partial \theta(z, t)}{\partial t}, \quad u_{, z}=\frac{\partial u(z, t)}{\partial z},
$$

а функции

$$
\mathscr{G}(\theta)=K_{1} \sin ^{2} \theta+K_{3} \cos ^{2} \theta
$$

и

$$
\mathcal{A}(\theta)=\frac{1}{2}\left(\gamma_{1}-\gamma_{2} \cos 2 \theta\right)
$$

отражают суть упругих и вязких сил соответственно. В этих выражениях $K_{1}$ и $K_{3}$ - упругие постоянные Франка, зависящие от температуры и соответствующие поперечным и продольным модам деформации, а $\gamma_{1}$ и $\gamma_{2}$ - коэффициенты вращательной вязкости нематика, которые также зависят от температуры. При этих условиях уравнение баланса моментов, действующих на единицу объема ЖК-ячейки, принимает вид [8]

$$
\begin{aligned}
\gamma_{1} \theta_{, t}= & \mathcal{A}(\theta) u_{, z}+\mathscr{G}(\theta) \theta_{, z z} \\
& +\frac{1}{2} \mathscr{G}_{\theta}(\theta) \theta_{, z}^{2}-\xi \theta_{, z} T_{, z}\left(\frac{1}{2}+\sin ^{2} \theta\right),
\end{aligned}
$$


а условие баланса импульсов, действующих на единицу объема ЖК-ячейки, позволяет записать аналог уравнения Навье-Стокса для нематической фазы в виде [8]

$$
\rho_{m} u_{, t}(z, t)=\sigma_{z x, z},
$$

где

$$
\begin{aligned}
\sigma_{z x} & =\frac{\delta \mathscr{R}}{\delta u_{, z}} \\
& =h(\theta) u_{, z}-\mathscr{A}(\theta) \theta_{, t}-\xi T_{, z} \theta_{, z} \sin ^{2} \theta\left(1+\frac{1}{2} \sin ^{2} \theta\right)
\end{aligned}
$$

- тангенциальная составляющая тензора напряжений $\sigma_{i j}, \mathscr{R}=\mathscr{R}_{v i s}+\mathscr{R}_{t m}+\mathscr{R}_{t h}-$ полная диссипационная функция Рэлея [8,14],

$$
\begin{gathered}
\mathscr{R}_{v i s}=\frac{1}{2} h(\theta) u_{, z}^{2}-\mathcal{A}(\theta) \theta_{, t} u_{, z}+\frac{1}{2} \gamma_{1} \theta_{, t}^{2}, \\
\mathscr{R}_{t m}=\xi \theta_{, t} \theta_{, z} T_{, z}\left(\frac{1}{2}+\sin ^{2} \theta\right) \\
-\xi T_{, z} u_{, z} \theta_{, z} \sin ^{2} \theta\left(1+\frac{1}{2} \sin ^{2} \theta\right), \\
\mathscr{R}_{t h}=\frac{1}{2 T}\left(\lambda_{\|} \cos ^{2} \theta+\lambda_{\perp} \sin ^{2} \theta\right) T_{, z}^{2}, \\
h(\theta)=\alpha_{1} \sin ^{2} \theta \cos ^{2} \theta-\mathcal{A}(\theta) \theta_{, t} u_{, z}+\frac{1}{2} \alpha_{4}+g(\theta), \\
g(\theta)=\frac{1}{2}\left(\alpha_{6} \sin ^{2} \theta+\alpha_{5} \cos ^{2} \theta\right),
\end{gathered}
$$

$\alpha_{i}(i=1, \ldots, 6)$ - шесть коэффициентов Лесли, $\lambda_{\|}$ и $\lambda_{\perp}-$ коэффициенты теплопроводности ЖК-фазы в направлении параллельном и перпендикулярном направлению директора $\hat{\mathbf{n}}$.

В случае зарождения градиента температуры по сечению ГОЖК-канала поле температуры $T(z, t)$ должно удовлетворять уравнению теплопроводности $[8,15]$

$$
\rho_{m} C_{p} T_{, t}=-q_{z, z},
$$

где $C_{p}$ - теплоемкость ЖК-системы, а $q_{z}=-T \frac{\delta \mathscr{R}}{\delta T_{z}}-$ тепловой поток в ГОЖК-канале. Принимая во внимание тот факт, что $\mathbf{v}=u(z, t) \hat{\mathbf{i}}$ и $\hat{\mathbf{n}}=(\sin \theta, 0, \cos \theta)$, последнее уравнение может быть записано в виде $[8,15]$

$$
\begin{aligned}
& \rho_{m} C_{p} T_{, t}=\lambda_{\perp}\left[T_{, z}\left(\lambda \cos ^{2} \theta+\sin ^{2} \theta\right)\right]_{, z} \\
& +\left[\xi T \theta_{, z}\left(\theta_{, t}\left(\frac{1}{2}+\sin ^{2} \theta\right)-u_{, z} \sin ^{2} \theta\left(1+\frac{1}{2} \sin ^{2} \theta\right)\right)\right]_{, z},
\end{aligned}
$$

где $\lambda=\lambda_{\|} / \lambda_{\perp}$. Для того чтобы провести анализ полученной системы уравнений (6), (7) и (9), перепишем ее в безразмерном виде

$$
\begin{gathered}
\theta_{, \tau}=\overline{\mathscr{A}}(\theta) u_{, z}+\overline{\mathscr{G}}(\theta) \theta_{, z z} \frac{1}{2} \overline{\mathscr{G}}_{, \theta}(\theta) \theta_{, z}^{2} \\
-\delta_{1} \chi_{, z} \theta_{, z}\left(\frac{1}{2}+\sin ^{2} \theta\right), \\
\delta_{2} u(z, \tau)_{, \tau}=\bar{\sigma}_{z x, z},
\end{gathered}
$$

и

$$
\begin{aligned}
& \delta_{3} \chi(z, \tau)_{, \tau}=\left[\chi, z\left(\lambda \cos ^{2} \theta+\sin ^{2} \theta\right)\right]_{, z}+\delta_{4} \\
& \times\left[\chi \theta_{, z}\left(\theta_{, \tau}\left(\frac{1}{2}+\sin ^{2} \theta\right)-u_{, z} \sin ^{2} \theta\left(1+\frac{1}{2} \sin ^{2} \theta\right)\right)\right]_{, z},
\end{aligned}
$$

где

$$
\begin{aligned}
\bar{A}(\theta) & =\mathscr{A}(\theta) / \gamma_{1}, \quad \overline{\mathscr{G}}(\theta)=\mathscr{G}(\theta) / K_{1}, \quad \bar{\sigma}_{z x}=\sigma_{z x} / \gamma_{1}, \\
\sigma_{z x} & =\frac{\delta \mathscr{R}}{\delta u_{, z}} \\
& =h(\theta) u_{, z}-\mathscr{A}(\theta) \theta_{, \tau}-\xi T_{, z} \theta_{, z} \sin ^{2} \theta\left(1+\frac{1}{2} \sin ^{2} \theta\right)
\end{aligned}
$$

- сдвиговая компонента тензора напряжений. В уравнении (12) $\chi(\tau, z)=T(\tau, z) / T_{N I}$ - безразмерная температура нормированная на температуру фазового перехода $T_{N I}$ нематик-изотропное состояние, $\tau=\left(K_{1} / \gamma_{1} d^{2}\right) t-$ безразмерное время, $z=z / d-$ безразмерная пространственная переменная отсчитанная от нижней границы ЖК-канала, в то время как $\delta_{1}=\xi T_{N I} / K_{1}, \delta_{2}=\rho_{m} K_{1} / \gamma_{1}^{2}$, $\delta_{3}=\rho_{m} C_{p} K_{1} /\left(\gamma_{1} \lambda_{\perp}\right) \quad$ и $\delta_{4}=K_{1} \xi /\left(\gamma_{1} \lambda_{\perp} d^{2}\right)$ - четыре параметра ЖК-системы. Здесь и далее под $z$ будем подразумевать $z / d$. Система безразмерных уравнений (10)-(12) должна быть дополнена граничными условиями как для поля директора и скорости, так и для поля температуры. Безразмерное граничное условие для поля директора принимает вид

$$
\theta_{z=0}=\frac{\pi}{2}, \quad \theta_{z=1}=\pi,
$$

в то время как безразмерное граничное условие для поля скорости может быть записано в виде

$$
u(z)_{z=0}=u(z)_{z=1}=0 .
$$

В свою очередь, безразмерное поле температуры $\chi(z, \tau)$ должно удовлетворять граничному условию

$$
\chi(z)_{z=0}=\chi_{1}, \quad(\partial \chi(z) / \partial z)_{z=1}=0 .
$$

Таким образом, формирование градиента температуры по сечению ГОЖК-канала, в котором верхняя поверхность полностью термически изолирована, а на нижней поверхности поддерживается постоянная температура, под действием стационарного горизонтального гидродинамического потока описывается системой нелинейных дифференциальных уравнений в частных производных (10)-(12), дополненных граничными условиями (13)-(15).

В случае ЖК-системы, образованной молекулами 4- $n$-пентил- $n^{\prime}$-цианобифенила (5ЦБ), температурный интервал существования нематической фазы соответствует $295 \leq T \leq T_{N I} \sim 307 \mathrm{~K}$. В этом интервале температур среднее значение плотности нематической фазы равно 
$\sim 10^{3} \mathrm{~kg} / \mathrm{m}^{3}$, средние значения коэффициентов упругости $K_{1}$ и $K_{3}$ равны 10 и $13 \mathrm{pN}$ [16] соответственно, в то время как среднее значение коэффициента вращательной вязкости $\gamma_{1}$ равно $0.072 \mathrm{~Pa} \cdot \mathrm{s}$ [17]. Значения коэффициентов теплоемкости и теплопроводности в этом температурном интервале в среднем постоянны и равны $C_{p} \sim 10^{3} \mathrm{~J} / \mathrm{kg} \cdot \mathrm{K}[18]$ и $\lambda_{\|} \sim 0.24$ и $\lambda_{\perp} \sim 0.13 \mathrm{~W} / \mathrm{m} \cdot \mathrm{K}[19]$ соответственно. С учетом значений коэффициентов Лесли, соответствующих нематической фазе 5ЦБ [20], величины параметров $\delta_{i}$ $(i=1, \ldots, 4)$ равны $\delta_{1} \sim 24, \delta_{2} \sim 2 \cdot 10^{-6}, \delta_{3} \sim 6 \cdot 10^{-4}$, $\delta_{4} \sim 210^{-9}$ соответственно. Поэтому с учетом того, что $\delta_{i} \ll 1 \quad(i=2,3,4)$, уравнения Навье-Стокса (11) и теплопроводности (12) упрощаются. Так, уравнение (11) принимает вид

$$
\begin{aligned}
\bar{\sigma}_{z x} & =h(\theta) u_{, z}-\mathcal{A}(\theta) \theta_{, \tau}-\delta_{1} \chi_{, z} \theta_{, z} \sin ^{2} \theta\left(1+\frac{1}{2} \sin ^{2} \theta\right) \\
& =C(\tau),
\end{aligned}
$$

где $C(\tau)$ - функция не зависящая от пространственной переменной $z$ и определяющаяся граничными условиями, а уравнение теплоповодности (12) принимает вид

$$
\left[\chi_{, z}\left(\lambda \cos ^{2} \theta+\sin ^{2} \theta\right)\right]_{, z}=0 .
$$

С целью изучения механизма ответственного за формирование градиента температуры по сечению ГОЖК-канала под действием стационарного горизонтально направленного гидродинамического потока рассмотрим стационарный аналог уравнения (10), где $\theta_{, \tau}=0$. В этом случае безразмерное температурное поле $\chi(z)$ в ГОЖК-канале описывается уравнением

$$
\chi(z)=\int_{0}^{z}\left(h(\theta) u_{, z}-\left(h(\theta) u_{, z}\right)_{z=1}\right) / I(\theta, z) d z+\chi_{1},
$$

где

$$
I(\theta, z)=\delta_{1} \theta_{, z} \sin ^{2} \theta\left(1+\frac{1}{2} \sin ^{2} \theta\right)
$$

и $\chi_{1}=T_{1} / T_{N I}$.

\section{3. Решение гидродинамических уравнений и основные результаты}

Формирование разности температур по сечению изначально равномерно прогретого ГОЖК-канала под действием стационарного горизонтально направленного гидродинамического потока, с острым „треугольным“ профилем

$$
u(z, \zeta)= \begin{cases}\frac{\alpha z}{\xi}, & (0 \leq z<\zeta) \\ \frac{\alpha}{1-\xi}(1-z), & (\xi \leq z<1)\end{cases}
$$

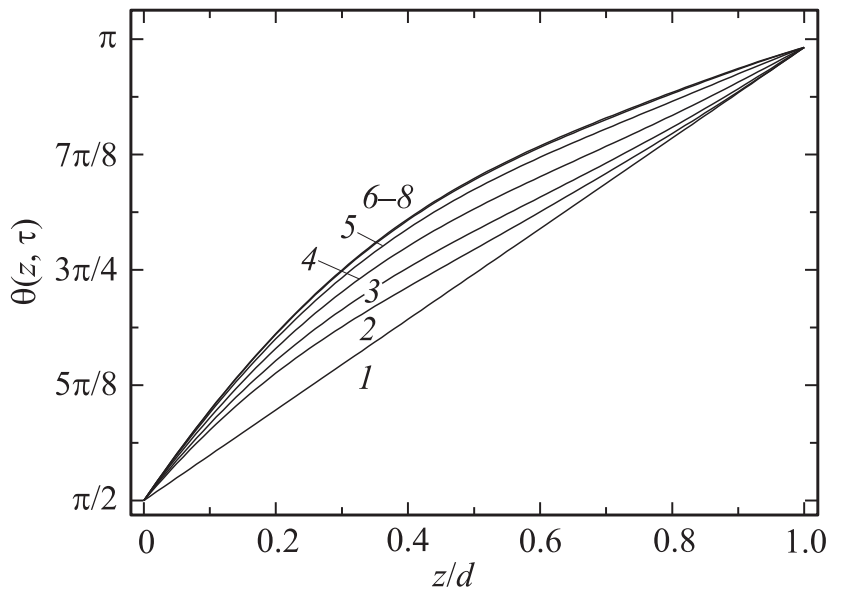

Рис. 1. Релаксация полярного угла $\theta(z, \tau)$ к равновесному распределению $\theta_{\text {eq }}(z)$ по сечению гибридно-ориентированного ЖК-канала. Кривая 1 соответствует значению $\tau=0.004$, кривая $8-$ равновесному распределению спустя $\tau_{R}=\tau(8)=0.032(\sim 23 \mathrm{~ms})$. Кривые $2-7$ соответствуют промежуточным значениям $\tau$.

было исследовано численно методом релаксации [21], а критерием сходимости итерационной процедуры был выбран $\epsilon=\left|\left(\theta_{m+1}(z, \tau)-\theta_{m}(z, \tau)\right) / \theta_{m}(z, \tau)\right| \sim 10^{-4}$. Здесь $m-$ номер итерации. Этот профиль гидродинамической скорости характеризуется двумя параметрами, положением $\xi$ и величиной $\alpha$ максимального значения скорости $u(z, \zeta)$.

Как уже было отмечено выше, при отсутствии потока в ГОЖК-канале устанавливается линейное $\theta(z)=\frac{\pi}{2}(z+1)$ распределение поля директора по сечению ЖК-канала, так, что на нижней границе $\theta_{z=0}=\frac{\pi}{2}$ и директор соориентирован планарно, в то время как на верхней границе $\theta_{z=1}=\pi$ и директор соорентирован гомеотропно. Как только в ГОЖК-канале формируется горизонтально направленный поток $u(z, \xi)$, то происходит переориентация поля директора и распределение угла $\theta(z, \tau)$ эволюционирует к его равновесному распределению $\theta_{\mathrm{eq}}(z)$ по сечению ЖК-канала. Результаты расчета эволюции $\theta(z, \tau)$ к равновесному значению $\theta_{\text {eq }}\left(z, \tau_{R}=\tau(8)\right)$ представлены на рис. 1. Здесь $\tau_{R}=\tau(8)=0.032(\sim 23 \mathrm{~ms})$, а величина параметра $\alpha$ равна 0.09. Это соответствует размерному значению максимальной скорости в ГОЖК-канале шириной в $10 \mu \mathrm{m}$, в $\sim 0.9 \mu \mathrm{m} / \mathrm{s}$, приложенной в точке $\xi=0.98$. Равновесный профиль полярного угла $\theta_{\mathrm{eq}}\left(z, \tau_{R}=\tau(8)\right)$, отсчитанного от оси $z$, характеризуется нелинейной зависимостью от расстояния $z$, и все итерационные кривые $\theta\left(z, \tau_{i}\right)$ располагаются выше прямой $\theta(z)=\frac{\pi}{2}(z+1)$. Такое поведение $\theta\left(z, \tau_{i}\right)$ физически обосновано, поскольку гидродинамический поток сильнее отклоняет директор в середине ЖК-канала чем вблизи границ, где директор жестко соориентирован. В свою очередь, как только в ГОЖК-канале формируется гидродинамический поток $u(z, \xi)$ и происходит переориентация 


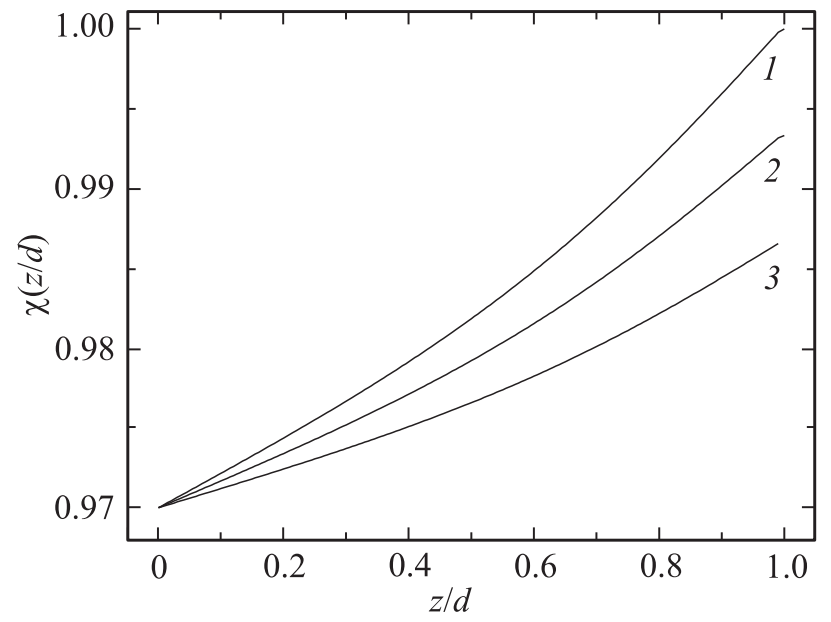

Рис. 2. Зависимость безразмерной температуры $\chi(z / d)$ от безразмерного расстояния $z / d$, отсчитанного от нижней ограничивающей поверхности ГОЖК-канала. Параметры гидродинамической скорости $u(z, \xi)$ равны: $\alpha=0.09$ (кривая 1 ), 0.07 (кривая 2), 0.05 (кривая 3) соответственно. Во всех случаях $\xi=0.98$.

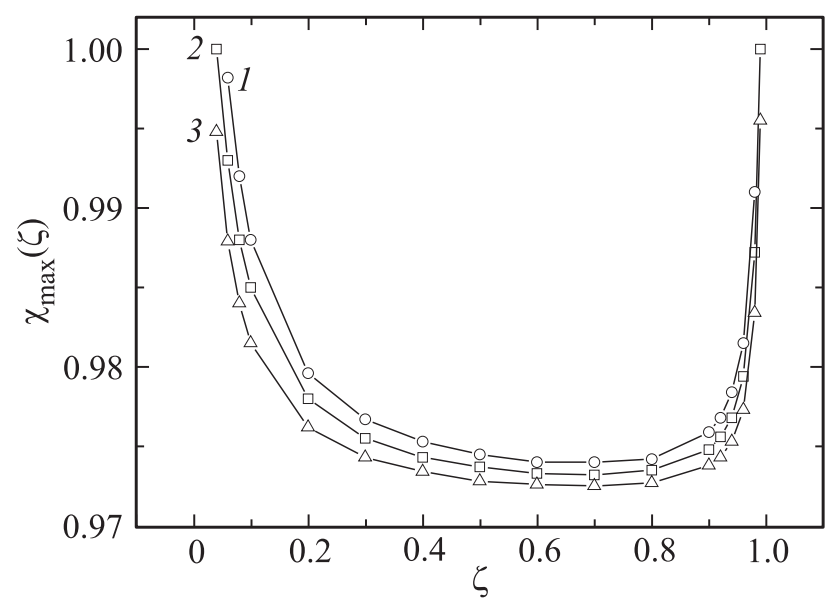

Рис. 3. Зависимость $\chi_{\max }(\xi)$ от положения $\xi$ максимального значения скорости $u(z, \xi)$ для трех значений $\alpha: 0.11$ (кривая 1), 0.09 (кривая 2), 0.07 (кривая 3).

поля директора, по сечению ГОЖК-канала начинает формироваться разность температур $\Delta \chi=\chi_{\mathrm{up}}-\chi_{\mathrm{lw}}$. Это обусловлено термомеханической силой, которая дает вклад как в выражение для полной диссипационной функции Рэлея $\left(\mathscr{R}_{t m}\right)$, так и в баланс моментов $\left(\mathbf{T}_{t m}\right)$, действующих на единичный объем ЖК-фазы. Следует отметить, что в изотропной фазе термомеханический вклад отсутствует, поскольку отсутствует поле директоpa. Когда стационарный поток $\mathbf{v}=u(z, \xi) \hat{\mathbf{i}}$ направлен в положительном направлении (см. рис. 2), температура на нижней границе ГОЖК-канала поддерживается постоянной $\chi_{z}=0=\chi_{1}=0.97(\sim 298 \mathrm{~K})$, в то время как по сечению ЖК-канала формируется температурный градиент $\nabla T$, направленный к более теплой верхней границе ГОЖК-канала. Максимальная температурная разница $\chi_{\max }(\xi) \equiv \Delta \chi=\chi_{\text {up }}-\chi_{\mathrm{lw}}=0.03(\sim 9 \mathrm{~K})$ формируется в ГОЖК-канале под влиянием стационарного потока $u(z, \xi)$, когда величина параметра $\alpha$ равна 0.09 (кривая (1)), чему соответствует размерное значение максимальной скорости в ГОЖК-канале шириной в $10 \mu \mathrm{m}$, в $\sim 0.9 \mu \mathrm{m} / \mathrm{s}$, приложенное в точке $\xi=0.98$. Для двух других режимов, с $\alpha=0.07$ и 0.05 , максимальная температурная разница $\chi_{\max }(\xi)$ равна $0.02(\sim 6 \mathrm{~K})$ и $0.01(\sim 3 \mathrm{~K})$ соответственно. Во всех этих трех случаях положение максимального значения гидродинамической скорости $\xi=0.98$ было вблизи верхней гомеотропно ориентированной поверхности ЖК-канала. Следует отметить, что такой выбор параметров $\alpha$ и $\xi$ был предопределен небольшим интервалом $\chi \in[0.97-1.00]$ существования нематической фазы. Было также установлено, что положение $\xi$ максимального значения скорости $u(z, \xi)$ сильно влияет на величину максимальной разности температур $\chi_{\max }(\xi)$, формирующейся по сечению ГОЖК-канала (см. рис. 3). Так, например, в случае $\xi=0.5$, т.е. когда положение максимального значения гидродинамической скорости находилось в середине сечения ЖК-канала, температурная разница $\chi_{\max }(\xi=0.5)=0$ и градиент температуры поперек ГОЖК-канала не формировался. Даже увеличив максимальное значение скорости $\alpha$ на один порядок, с 0.09 до 0.9 , температурная разность $\chi_{\max }(\xi=0.5) \sim 0.001$ оставалась практически равной нулю. С другой стороны, когда положение $\xi$ максимального значения скорости $u(z, \xi)$ находится вблизи ограничивающих поверхностей, тогда и достигается максимальная разность температур по сечению ГОЖК-канала. Такая зависимость $\chi_{\max }(\xi)$ от $\xi$ предопределена характером уравнения (18) и „треугольным“ профилем гидродинамической скорости по сечению ЖК-канала. Действительно, когда $\xi \rightarrow 0$ или 1 , то максимальная величина $u(z, \xi)$ неограниченно возрастает и тем самым формируется большая разность температур $\chi_{\max }(\xi)$. Следует также отметить, что наш выбор положения $\xi$ максимального значения скорости $u(z, \xi)$ был предопределен узким температурным интервалом существования нематической фазы, и выбор $0.05 \leq \alpha \leq 0.09$ был продиктован этим условием.

\section{4. Заключение}

Предложено теоретическое описание формирования градиента температуры в изначально равномерно прогретом гибридно-ориентированном жидкокристаллическом (ГОЖК) канале микроскопических размеров под действием стационарного, горизонтально направленного гидродинамического потока. Численные исследования, выполненные в рамках нелинейного обобщения теории Эриксена-Лесли, с учетом уравнения теплопроводности, показали, что в случае полной термической изоляции одной из поверхностей ГОЖК-канала и при условии, что на другой поверхности поддерживается 
постоянная температура, под действием стационарного гидродинамического потока с „треугольным“ профилем скорости $u(z, \xi)$ возможно формирование разности температур $\chi_{\max }(\xi)=\chi_{\text {up }}-\chi_{\mathrm{lw}}$ по сечению ГОЖК-канала. На величину $\chi_{\max }(\xi)$ сильно влияет положение $\xi$ максимального значения скорости $u(z, \xi)$. Было показано, что в случае ЖК-системы, образованной молекулами 4- $n$-пентил- $\boldsymbol{n}^{\prime}$-цианобифенила, гидродинамический поток, характеризующийся „треугольным“ профилем скорости с $\xi=0.98$ и $u(z, \xi=0.98) \sim 0.09$, формирует максимальную разность температур $\chi_{\max }(\xi)=0.03(\sim 9 \mathrm{~K})$. Такой выбор положения максимального значения безразмерной скорости $u_{\max }(z, \xi=0.98)=0.09$ был предопределен узким температурным интервалом существования нематической фазы. Эта безразмерная величина скорости соответствует значению размерной максимальной скорости $\sim 0.9 \mu \mathrm{m} / \mathrm{s}$ в ГОЖК-канале шириной в $10 \mu \mathrm{m}$, приложенной в точке $\xi=0.98$. Следует отметить, что такая зависимость $\chi_{\max }(\xi)$ от $\xi$ предопределена также характером уравнения описывающего формирование поля температуры и формой „треугольного“ профиля гидродинамической скорости по сечению ЖК-канала. Описанный механизм диссипации механической энергии определяется корреляцией градиентов поля директора и температуры; эта корреляция ответственна за формирование разности температур поперек ГОЖК-канала под действием стационарного гидродинамического потока с „треугольным“ профилем скорости. Следует также отметить, что в однородно ориентированном ЖК-канале, где отсутствует градиент поля директора $\nabla \hat{\mathbf{n}}$, под действием гидродинамического потока с любым профилем скорости градиент температуры не формируется. Таким образом, этот механизм формирования градиента температуры в ЖК-канале реализуется только лишь под действием гидродинамического потока со специфическим профилем скорости и при условии существования градиента поля директора. В заключение также следует отметить, что из-за микроскопических размеров ЖК-канала в нем не может наблюдаться конвективная неустойчивость, обусловленная механизмом Рэлея-Бенара, поскольку величина параметра $R \sim d^{3}$, отвечающего за возникновение этой неустойчивости, значительно меньше универсального критического значения $R_{c} \sim 1708$ [8], определяющего порог, выше которого и начинает формироваться конвективная неустойчивость.

\section{Список литературы}

[1] R.B. Schoch, J.Y. Han, P. Renaud. Rev. Mod. Phys. 80, 839 (2008).

[2] A. Sengupta, U. Tkalec, M. Ravnik, J. Yeomans, Ch. Bahr, S. Herminhaus. Phys. Rev. Lett. 110, 048303 (2013).

[3] D.P. Holmes, B. Tavakol, G. Frochlicher, H.A. Stone. Soft. Matter. 9, 7049 (2013).

[4] A. Sugimura, A.V. Zakharov. Phys. Rev. E 84, 021703 (2011).

[5] A.V. Zakharov, A.A. Vakulenko. J. Chem. Phys. 139, 244904 (2013).
[6] Р.С. Акопян, Б.Я. Зельдович. ЖЭТФ 87, 1660 (1984).

[7] R.S. Akopyan, R.B. Aloverdian, E.A. Santrosian, Y.S. Chilingarian. J. Appl. Phys. 90, 3371 (2001).

[8] A.V. Zakharov, A.A. Vakulenko. J. Chem. Phys. 127, 084907 (2007).

[9] A.V. Zakharov, A.A. Vakulenko. RSC Advances. 2, 7296 (2012).

[10] O. Lehmann. Ann. Phys. 4, 649 (1900).

[11] J.L. Ericksen. Arch. Ration. Mech. Anal. 4, 231 (1960).

[12] F.M. Leslie. Arch. Ration. Mech. Anal. 28, 265 (1968).

[13] P.G. de Gennes, J. Prost. The physics of liquid crystals. Oxford Univ. Press., Oxford (1995). 400 p.

[14] I.W. Stewart. The static and dynamic continuum theory of liquid crystals. Taylor and Francis, London (2004). 345 p.

[15] Л.Д. Ландау, Е.М. Лифшиц. Гидродинамика. Наука, М. (1986). $736 \mathrm{c}$.

[16] A.V. Zakharov, A. Maliniak. Eur. Phys. J. E 4, 85 (2001).

[17] A.V. Zakharov, A.V. Komolkin, A. Maliniak. Phys. Rev. E 59, 6802 (1999).

[18] P. Jamee, G. Pitsi, J. Thoen. Phys. Rev. E66, 021707 (2002).

[19] M. Marinelli, A.K. Ghosh, F. Mercuri. Phys. Rev. E 63, 061713 (2001).

[20] A.G. Chmielewski. Mol. Chyst. Liq. Cryst. 132, 339 (1986).

[21] И.С. Березин, Н.Р. Жидков. Методы вычислений. Физматгиз, М. (1964). 464 с. 